\title{
Labyrinthe
}

$10 \mid 2001$

Numéro 10

\section{Paris, Berlin : la mémoire de la Première Guerre mondiale (1914-1933)}

\section{Élise Julien}

\section{(2) OpenEdition \\ 1 Journals}

\section{Édition électronique}

URL : http://journals.openedition.org/labyrinthe/1190

DOI : $10.4000 /$ labyrinthe.1190

ISSN : 1950-6031

\section{Éditeur}

Hermann

\section{Édition imprimée}

Date de publication : 1 octobre 2001

Pagination : 101-103

\section{Référence électronique}

Élise Julien, «Paris, Berlin : la mémoire de la Première Guerre mondiale (1914-1933) », Labyrinthe [En ligne], 10 | 2001, mis en ligne le 03 avril 2006, consulté le 22 avril 2019. URL : http://

journals.openedition.org/labyrinthe/1190; DOI : 10.4000/labyrinthe.1190

\section{Ce document a été généré automatiquement le 22 avril 2019}

Propriété intellectuelle 


\title{
Paris, Berlin : la mémoire de la Première Guerre mondiale (1914-1933)
}

\author{
Élise Julien
}

1 L'histoire pratique des approches multiples, dont le croisement est souvent fécond. Ce projet d'une recherche consacrée à la mémoire de la Première Guerre mondiale telle qu'elle s'est forgée à Paris et à Berlin entre 1914 et 1933 se situe pour sa part à la jonction de l'histoire de la mémoire, de l'histoire de la Première Guerre mondiale, de l'histoire urbaine et de l'histoire comparée".

2 Alors que le thème de la mémoire mobilise particulièrement l'historiographie contemporaine $^{1}$, cette recherche devra s'aider des réflexions récentes sur la question pour étudier la mémoire de la Première Guerre mondiale. Par mémoire, on entend ici celle que les contemporains se sont forgée pendant la guerre d'abord, puis dans les années d'après-guerre jusqu'en 1933. Cette période relativement réduite et cohérente doit permettre de saisir de manière dynamique l'émergence, la mise en place et l'évolution du souvenir de la guerre dans ces années, avant son instrumentalisation à outrance par les nazis.

3 Une telle approche historienne de la mémoire rend quasiment inévitables certains emprunts à la terminologie du sociologue Maurice Halbwachs. La notion de cadres sociaux de la mémoire ${ }^{2}$, en particulier, pourra aider à comprendre comment les souvenirs individuels peuvent recevoir une orientation propre à un groupe. On conçoit alors l'intérêt d'une étude de la mémoire pour appréhender des constructions d'identités : l'identité repose sur la mémoire, qui permet de se penser identique dans le temps ; la mémoire s'appuie inversement sur l'identité, qui donne à chacun la conscience que tel enchaînement de séquences temporelles a un sens pour lui-même. Il est difficile de savoir, parmi les multiples identités d'un individu, laquelle prime dans sa perception et son comportement à un moment précis; le travail d'appropriation de la mémoire, même nationale, s'accomplit cependant presque toujours dans le concret du quotidien, par la médiation de groupes, de situations et à des niveaux d'agrégat plus réduits que la nation. 
4 De ce point de vue, la ville constitue un niveau d'agrégat extrêmement intéressant. Objet relativement restreint en même temps que champ d'étude signifiant et cohérent, elle est un terrain privilégié pour établir des résultats pertinents concernant les expériences communes aux hommes. Toutes les villes n'ont cependant pas le même statut ni la même signification symbolique : l'importance des capitales demande qu'on leur prête une attention particulière. Leur caractère participe à la fois du local et du national ; il hésite souvent entre ces deux pôles. Il faudra ainsi étudier dans le détail comment la capitale, centre du pouvoir et formation sociale de taille considérable, parvient à se situer à la jonction de l'État national et de la société civile (définie comme l'ensemble des communautés quelles qu'elles soient), et comment elle peut devenir à la fois le symbole d'une mémoire nationale et le cadre d'une mémoire locale.

5 Si cette étude comparative ${ }^{3}$ envisage deux « espaces de mémoire » relativement proches (les capitales de deux grands pays européens continentaux, ceux-là mêmes entre lesquels s'est déclenchée la guerre dont on étudie les conséquences), Paris et Berlin sont aussi deux métropoles aux personnalités bien différentes, où la guerre a été diversement vécue. Les mémoires parisiennes et berlinoises de la Première Guerre mondiale offrent néanmoins la possibilité d'une comparaison méthodique : on le constate particulièrement à la manière dont, par le deuil, elles traitent un des éléments essentiels sur lesquels elles portent, la mort d'une multitude d'hommes.

Les années qui suivent la Première Guerre mondiale s'avèrent particulièrement riches pour une telle analyse culturelle. La guerre a suscité de nombreuses mutations, elle laisse des traces profondes dans les têtes et les gestes, engendrant de nouvelles communautés d'expérience et de mémoire. Le choix de cette période particulière de l'histoire de l'Europe présente encore un intérêt : si l'étude des communautés urbaines en guerre permet de saisir les formes locales et diversifiées d'une mémoire dont les fondements sont alors remis en cause, la perspective comparative permet d'appréhender également, devant l'expérience commune qu'a pu constituer la guerre, une forme de mémoire sinon universelle, du moins transnationale.

7 L'historien de la mémoire doit surmonter la tension, sans doute féconde mais souvent incommode, que produit l'écartèlement, constitutif du domaine culturel, entre l'institué et le vécu. Aussi, pour saisir cet objet complexe qu'est la mémoire de la Première Guerre mondiale, à la fois celle que les institutions publiques et la société civile ont construite à l'intention des individus et celle que ceux-ci ont effectivement conservée, on aura recours à des sources d'origine et de forme variées. Les destins individuels se situent néanmoins dans des destins collectifs, que ce soit ceux des familles, des professions, des paroisses, des quartiers, des villes... D'une manière générale, c'est l'étude de ces groupes sociaux que l'on essaiera de privilégier, sans pour autant exclure d'intégrer à cette analyse des éléments concernant plus spécifiquement la mémoire intime des personnes.

8 Monuments aux morts, Kriegerdenkmäler ${ }^{4}$ : la langue française insiste ici sur la mort, tandis que la langue allemande choisit de rappeler les combattants. L'étude des sources (entre autres celle des monuments aux morts) dans les deux capitales devra montrer comment, au-delà des mots et des perspectives nationales, la construction de la mémoire a été partout complexe, multiple et souvent ambiguë.

9 À terme, on devrait pouvoir mettre en relief la diversité mentale des sociétés considérées en se fondant sur le critère de la mémoire. Étudier la mémoire devra finalement conduire à appliquer les règles les plus « positivistes » du métier d'historien, celles qui objectivent 
et socialisent le temps. Cette histoire pourra alors révéler sa pleine dimension culturelle : le fait historique est toujours médiatisé ; le temps, dans sa durée, n'est pas une quantité mesurable, infiniment sécable ; porté par des hommes assemblés, il prend toujours au passage une coloration émotionnelle, porteuse de mémoire potentielle, qui donne de l'épaisseur humaine à la recherche historique sur la mémoire.

\section{NOTES}

*. Cet article expose un projet de recherche pour le doctorat en histoire sociale et culturelle contemporaine, et fait suite à la rédaction en 1999 d'un mémoire de DEA sur le sujet, sous la direction de Jean-Louis Robert (Université Paris I).

1. Voir en premier lieu Pierre Nora, «Entre histoire et mémoire », dans Les Lieux de mémoire, volume I, Paris, 1984.

2. Maurice Halbwachs, Les Cadres sociaux de la mémoire, Paris, 1925.

3. Cette perspective comparative permet d'intégrer une telle étude de Paris et Berlin au début du siècle au programme de recherche international mené par les professeurs JeanLouis Robert (Paris) et Jay Winter (Cambridge) sur les capitales européennes en guerre. Voir Capital Cities at War, Cambridge, 1997.

4. Kriegerdenkmal est le plus usité, mais les mots allemands que l'on peut traduire en français par « monument aux morts » sont nombreux : Ehrenmal insiste sur l'honneur, Gedächtnismal sur le souvenir, Mahnmal sur le rappel, Gedenkstätte sur la commémoration... Aucun n'évoque explicitement la mort. 